\title{
A wireless sensor network DV-Hop localization algorithm
}

\author{
Huamin Yang ${ }^{1, a}$,Qi Wang ${ }^{2, b}$,zhuang Liu ${ }^{3, c}$ \\ ${ }^{1}$ School of Computer Science and Technology, Changchun University of Science and Technology, \\ Changchun 130022, Jilin, China \\ ${ }^{2}$ School of Computer Science and Technology, Changchun University of Science and Technology, \\ Changchun 130022, Jilin, China \\ ${ }^{3}$ School of Computer Science and Technology, Changchun University of Science and Technology, \\ Changchun 130022, Jilin, China \\ ayhm@cust.edu.cn, ${ }^{\mathrm{b}}$ wangqi19880816@163.com, ${ }^{\mathrm{c}} 1164913626 @ q q . c o m$
}

Keywords: Wireless networks; DV-Hop; MATLAB; positioning accuracy

\begin{abstract}
This paper first introduces wireless sensor networks self-positioning classical algorithm DV-Hop (distance vector-hop) algorithm, then analyze its shortcomings, Finally, a new improved algorithm and through improved algorithm matlab simulation verify the situation after the positioning accuracy.Comparative analysis of experimental data, the improved algorithm error is smaller than the original algorithm, more precise positioning.
\end{abstract}

\section{Introduction}

In recent years, with the continuous development of a number of high-tech, multi-intersection of science has become a hot research. MEMS, wireless communications, embedded systems and sensor technology such as technology continues to evolve and become more mature, and promote the generation of wireless sensor networks and development. Wireless sensor network integrated embedded computing technology, modern network technology and distributed information processing technology and other advanced technology, It is these traditional disciplines of mutual integration and information technology achievements in the field of information science is a new direction of development ${ }^{[1]}$. Climate monitoring, the surrounding ambient temperature, light, humidity, etc. detection, atmospheric pollution monitoring, building structural integrity monitoring, home environment, unusual circumstances, airport or stadium chemical, biological threat detection and forecasting, etc., WSN would be an economical alternative to have broad application prospects.

As we all know, the sensor nodes to determine their location and the location of the incident for wireless sensor networks monitoring activities is crucial, but due to cost, power consumption, size and other factors, it is impossible that each node with GPS accuracy positioning capability ${ }^{[2]}$. In practice only a few nodes have accurate positioning capability. Therefore, the DV-Hop algorithm precise positioning for the future, monitor positioning with better help.

\section{DV-Hop algorithm}

Traditional DV-Hop algorithm ${ }^{[3]}$ positioning process is divided into three phases:

1) Unknown node and compute nodes each beacon minimum hops.

(1) Beacon nodes broadcast their locations to the neighbors of information packets, including the jump number field is initialized to 0 . Receiving node records to each beacon nodes having the minimum number of hops, ignoring a beacon node from the same large number of hops a packet. Then hop count plus one, and forwarded to the neighbors. Through this method, all nodes in the 
network to be able to record each beacon node under the minimum number of hops.

(2) Compute unknown node and beacon node's actual hop distance.

Each beacon nodes according to the first stage record other beacon nodes position information and the distance hops, using the equation (1) estimate the average hop actual distance.

2) Calculate and obtain the unknown node average hop distance. Beacon nodes by saving the coordinates of the other beacon nodes and the minimum number of hops using the equation (1) in the network calculate the average hop distance:

$$
c_{i}=\sum_{i \neq j} \frac{\sqrt{\left(x_{i}-x_{j}\right)^{2}-\left(y_{i}-y_{j}\right)^{2}}}{\sum_{i \neq j} h o p_{i j}}
$$

Among: $\left(x_{i}, y_{i}\right) 、\left(x_{j}, y_{j}\right)$ are the beacon coordinates of node $\mathrm{i}$ and $\mathrm{j}$, hop $_{i j}$ is a beacon nodes $\mathrm{i}$ and $\mathrm{j}$, the minimum number of hops between. Then, the beacon node will calculate the average distance per hop fields with a packet with a lifetime of broadcasting to the network, the unknown node record only received the first average distance of each jump, and forwarded to the neighbors. This strategy ensures that the most recent beacon node from the node receives the value of the average distance per hop. Unknown node receives the average hop distance, according to the recorded number of hops to each beacon node calculate the hop distance.

3 ) Using trilateration measurement or maximum likelihood estimation method to calculate its own position. Unknown node uses the second phase to each record jump distance beacon nodes using trilateration measurement or maximum likelihood estimation method to calculate their coordinates.

\section{DV-Hop algorithm analyzes the deficiencies}

(1) In the DV-Hop algorithm, taken between beacon nodes as the average distance per hop beacon nodes unknown node to the average distance per jumping, jumping distance and jumping through each multiplied by the number to represent the unknown nodes and beacon nodes the distance between. However, in actual network topology beacon node to the unknown node is often not a straight path, use the DV-Hop algorithm will bring a greater distance error ${ }^{[4]}$.

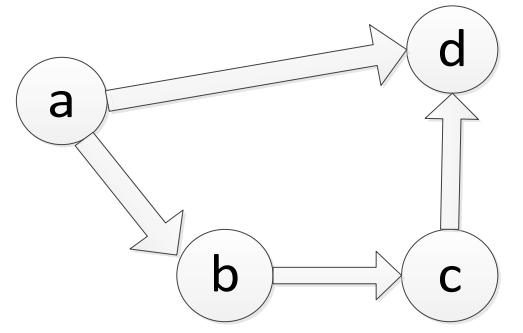

Fig. 1 node path schematically

Shown in Figure 1, take any four nodes a, b, c, d. Assuming the beacon node a node, the distance between the beacon nodes of $1 \mathrm{~m}$. Since DV-Hop algorithm, for unknown node d, a beacon nodes to the unknown node $\mathrm{d}$ is the distance $4 \mathrm{~m}$. However, this distance is much larger than the actual distance between two points, as the beacon node a to unknown node $d$ paths that are not straight connection. However, in practical wireless sensor network environment, the majority of the beacon nodes and unknown nodes are connected to form a polygonal line, Therefore, using the average hop distance and the product of the number of hops is defined as the distance of a beacon 
node to an unknown will cause large errors.

(2) DV-Hop localization algorithm assumes that the network average hop distance is the same, thereby reducing the positioning accuracy, especially when unknown beacon node under test and beacon node is one hop distance. In fact, one hop distance can be shorter or longer, not completely equal. And the beacon nodes for a single hop communication unknown nodes, the actual distance between them may be much smaller than or is much larger than the average hop distance, The DV-Hop algorithm average hop distance is instead of the actual distance between them, which will bring a larger positioning error.

\section{Improved DV-Hop algorithm}

\subsection{DV-Hop Exist improved algorithm}

For DV-Hop itself instead of the average distance of each average hop distance limitations. Literature [1] proposed a weighted average hop distance improved method .The set threshold, the unknown node accepts $\mathrm{M}$ hop within anchor nodes and weighted according to the hop count to calculate the integrated average hop distance. And literature [2] in consideration of the angle between the three adjacent nodes on the distance, and puts forward a set of adjacent nodes overlap angle calculation methods to improve the positioning accuracy. These two are from the original DV-Hop algorithm to calculate the average distance limitations to start, but have increased the unknown node to an anchor node distance complexity.

4.2 Concrete steps to improve the process described below:

(1) Broadcast packets anchor node and compute the distance between the anchor node and neighboring nodes.

Anchor nodes broadcast packets containing numbers, location coordinates, the number of hops and priority information. Ordering Priority equals 0 means that the first stage of anchor nodes, the highest priority. Because of this study taking into account the first non-distance ranging, and within one hop distance may be far less than the average distance, Based on the reference ranging $\mathrm{RSSI}^{[5]}$ approach within a hop distance of the measurement more accurate. When you want to locate unknown nodes and anchor nodes to one hop, the RSSI can directly calculate the distance between them and save. So within one hop gained the unknown node hops, and priority information, and the new position of these nodes can be used as the next anchor node, the number of effective expansion anchor node.

(2) Unknown node will get the information to upgrade to the new anchor node, Using trilateration measurement to fix its location, when the unknown nodes to obtain three or more of the anchor node information, through trilateration measurement ${ }^{[6]}$ to calculate the coordinates of its positioning. The node has been located as a new anchor node. First, the unknown node within hop upgraded to anchor node, While the newly generated priority anchors anchor node set lower than the original priority. Therefore, the new anchor node Priority equals 1.

The lower the priority value, the higher the priority. Low priority anchor nodes only in the high priority the number of anchor nodes are considered insufficient to complete the positioning when coming up as the reference node. And these new anchors also can be help to solve the previous positing problem. It should be noted that, if the number is less than three anchor nodes, the node can't locate the unknown, but can keep the node information to be used again when the next condition is satisfied, but also improve the location accuracy.

(3) Average hop distance estimates

First, from the unknown node with the nearest anchor node to each of the other anchor node 
distance divided by the minimum number of hops between them to get the average number of hops. And the obtained average hop distance and the original average hop distance to the arithmetic mean of the average hop distance instead of the original. Using equation (2) obtained more accurate average hop distance, closer to the actual position.

$$
D^{\prime}=\frac{D}{2}+\frac{d_{a b}}{2 h o p_{a b}}
$$

Among $D$ is the original average hop distance,$d_{a b}$ is the distance between beacon node a and

b. Hop $p_{a b}$ is the hops between anchor nodes, $D^{\prime}$ is the average hop for the final correction hops.

\subsection{Improved DV-Hop algorithm flowchart}

Shown in Figure 1,the improved DV-Hop algorithm with the original algorithm is slightly different in the beginning, in the anchor node adds priority information, so that the unknown node upgraded to known nodes. And then calculating the average hop distance optimization methods, the latter is substantially the same phase.

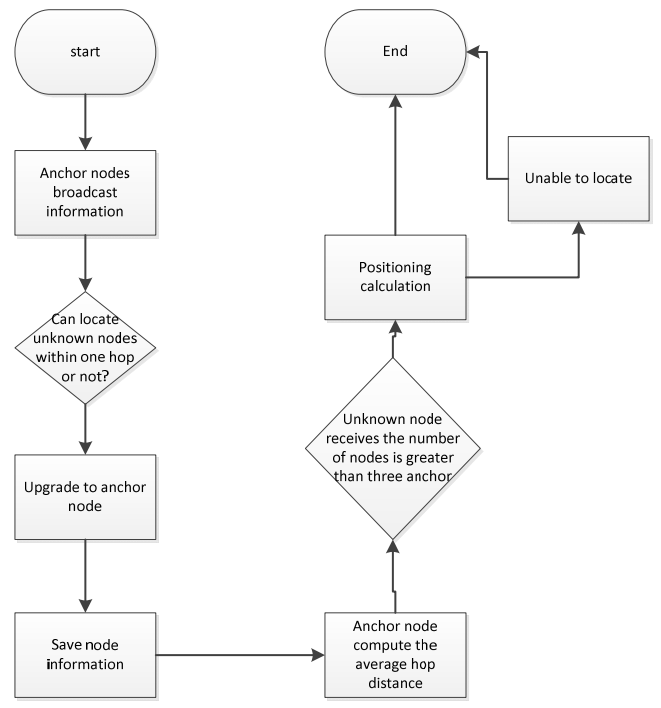

Fig.1 The improved algorithm flowchart

\section{Simulation and analysis of experimental results}

In this paper, the improved algorithm and MATLAB original algorithm simulation experiments, and the experimental results were analyzed and compared.

In the experiment on the assumption that the region of $100 * 100 \mathrm{~m}$ distribution sensor nodes is 20 , when the anchor of nodes is $10 \%$ of the total number of nodes, The distribution of each node shown in Figure 2,among o represents the anchor node, $\bullet$ represents the unknown node. 


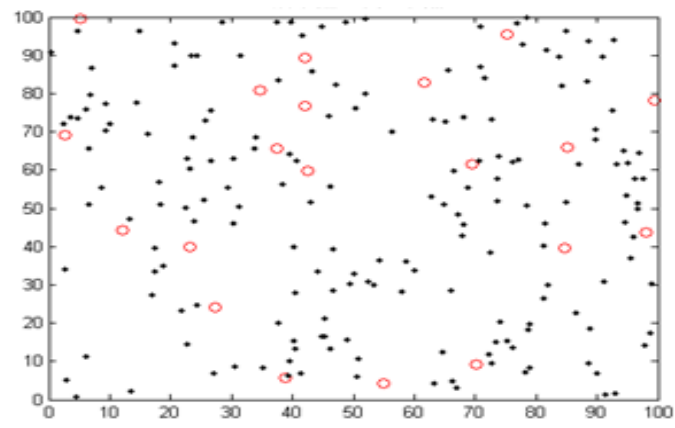

Figure 2 Anchor node and the positing node distribution chart

The improved algorithm and the original positioning DV-Hop algorithm overall contrast, as shown in Figure 3.

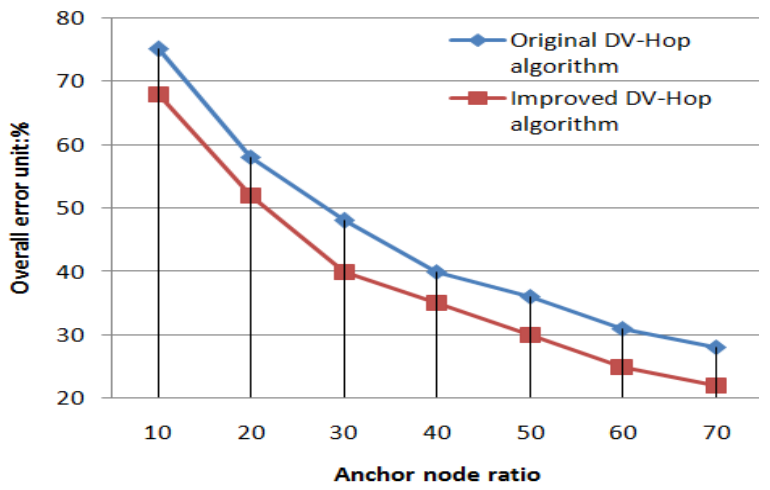

Fig. 3 The improved algorithm with the original DV-Hop algorithm location error comparison chart

Ranging improved compared in Figure 4.

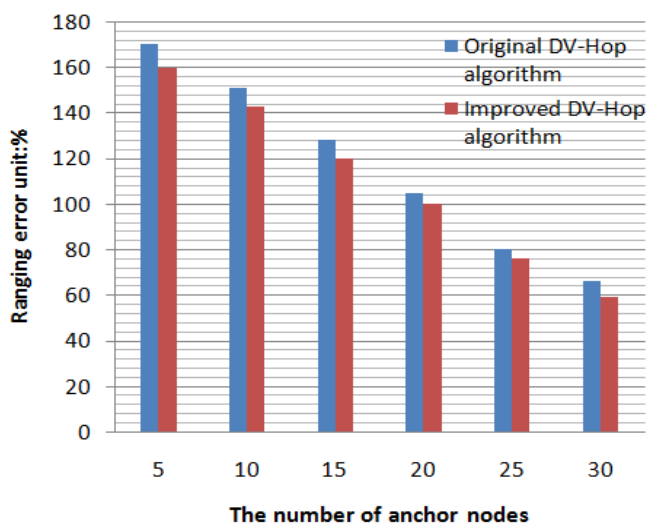

Fig. 4 The improved algorithm and the original algorithm as anchor nodes increases ranging error comparator 
Figure 3 shows that the proportion of the anchor node in the whole process, from start to finish the positioning accuracy is higher than the original DV-Hop algorithm. This not only shows the superiority of the improved algorithm, also makes the unknown node hop has a higher accuracy than the original DV-Hop algorithm similar node. While also expanding the anchor nodes.

Figure 4, with the anchor nodes increasing, the error rate of the two algorithms are reduced, however, the improved algorithm the error rate is lower, closer to the actual values.

\section{Summary}

This paper first introduces the original through the DV-Hop algorithm, pointing out its deficiencies, and analyzing the reasons for its deficiencies, as well as areas for improvement. Based on this, a new algorithm for DV-Hop algorithm, the broadcast data packet priority increasing, effectively to expanse the anchor nodes. Also optimizes network average hop distance estimates the new calculation of the average hop distance calculation is more accurate, realistic position than the original algorithm, effectively reduce the error, given the improved algorithm for DV-Hop flowchart . Finally, through matlab simulation experiments, analysis of the improved algorithm with the original algorithm positioning error and range error, confirming the improved algorithm greatly improved accuracy.

\section{References}

[1] $\mathrm{Hu} \mathrm{Yu}$, Li Xuemei based on DV-HOP algorithm for wireless sensor network node positioning technology. Shanxi: Taiyuan University of Technology, 2012,5

[2] Zhang Xiaolong, Xie Hui-ying wireless sensor networks in an improved DV-Hop localization algorithm Hunan: Wuhan University of Technology, 2008,3.

[3] D Niculescu, B Nath. Ad-Hoc Positioning System(APS)[J].IEEE GlobalTelecommunications, 2001, 5: 2926-2931.

[4] Zhang Xiaolong, Xie Hui-ying, Zhao Xiaojian wireless sensor networks in an improved DV-Hop localization algorithm [J]. Journal of Computer Applications, 2007,27 (11) :2672 -2674.

[5]YunWang,XiaodongWang,DeminWang,Agrawal,D.P.ARSSI-basedDV-hopAlgorlthimforWirele ssSensorNetworks[J].IEEETransaetions011ParallelandDistributedSystems,2009,20(10):1540.1552.

[6] Zhangshu Peng wireless sensor network positioning technology research [D]. Guangzhou: South China University of Technology, 2010.

[7]Zhuang Liu, Zhiyi Fang, Naiji Ren, Yang Zhao. “A New Range-Free Localization Algorithm Based on Annulus Intersection and Grid Scan in Wireless Sensor Networks”. Journal of Information and Computational Science. Vols 9, number 4, 2012. 831-841.

[8]Lin Chen, Zhiyi Fang, Wei Lv and Zhuang Liu. “An improved DV-HOP localization algorithm based on Simulation Curve Fitting”. Advanced Materials Research. Vol.648(2013) P390-393. 